\title{
Flight parameters improvement for an unmanned aerial vehicle using a lookup table based fuzzy PID controller
}

\author{
Abderrahmen Benbouali ${ }^{1}$, Fayçal Chabni' ${ }^{2}$ Rachid Taleb $^{3}$, Noureddine Mansour ${ }^{4}$ \\ 1,2,3Electrical Engineering Department, Hassiba Benbouali University of Chlef, \\ Laboratoire Génie Electrique et Energies Renouvelables (LGEER), Algeria \\ ${ }^{4}$ College of Engineering, University of Bahrain, Bahrain
}

\section{Article Info}

Article history:

Received Apr 2, 2021

Revised Jun 2, 2021

Accepted Jun 10, 2021

\section{Keywords:}

Fuzzy logic

Lagrange approach

PID controller

Quadrotor

STM32F407 microcontroller

\begin{abstract}
In this paper, a control scheme based on lookup table fuzzy proportionalintegral-derivate (PID) controller for the quadrotor unmanned aerial vehicle (UAV) movement control is proposed. This type of control provides enhanced quadrotor movement control beyond what can be achieved with conventional controllers and has a less computational burden on the processor. The proposed control scheme uses three lookup table based fuzzy logic controllers to control the different movement ranges of a quadrotor (i.e. roll, pitch, and yaw) to achieve stability. The mathematical model of a quadrotor, used to design the proposed controller, is derived based on the Lagrange approach. The processor in the loop (PIL) technique was used to test and validate the proposed control scheme. MATLAB/Simulink environment was used as a platform for the quadrotor model, whereas a low cost and high-performance STM32F407 microcontroller was used to implement the controllers. Data transfer between the hardware and software is via serial communication converter. The control system designed based on simulation is tested and validated using "processor in the loop" techniques.
\end{abstract}

This is an open access article under the CC BY-SA license.

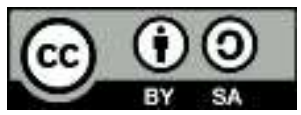

\section{Corresponding Author:}

Abderrahmen Benbouali

Electrical Engineering Departement

Hassiba Benbouali University of Chlef, Algeria

Laboratoire Génie Electrique et Energies Renouvelables (LGEER)

Email: abd.benbouali@gmail.com

\section{INTRODUCTION}

Unmanned aerial vehicles, or UAVs, have several advantages over conventional manned vehicles including an increased operational range, increased maneuverability; low production cost, and avoid putting personnel at risk [1]-[5]. All these advantages were incentives to attract the attention of the military community around the world in the improvement and application of these devices. UAVs are one of the most used vehicles in combat around the globe; they are utilized for intelligence gathering, surveillance, relaying communication (network node) and they can even be used as strike aircraft. With their ability to carry sensors, cameras, actuators, or even heavy loads, UAVs are also finding a way into the civilian market. For example, UAVs can be used to deliver vital supplies such as medicaments and communication devices in remote areas; they can also be used for monitoring pollution or crop inspection.

In recent years, scientists and researchers have developed multiple control strategies to enhance and improve the stability and movement of unmanned aerial systems [6], [7]. Most of these methods involve the use of conventional PID controllers [8]-[11] which are easy to implement. However, they are not recommended for applications requiring precision during maneuvers such as inspection drones carrying 
on-board cameras. This is due to poor performance such as presence of peaks and difficulty of disturbance rejection. Some other methods use complex control algorithms such as fuzzy logic controllers [12]-[16] which is good in performance but that require a lot of processing power. This paper proposes a new algorithm of control using a Lookup table based on Fuzzy PID (LFPID) controller to achieve enhanced stabilization. The distinguishing feature of the proposed algorithm is the use of a pre-calculated inference system to perform the various maneuvers of our aerial robot. Our method provides better performance than that of a conventional PID controller and it requires less computational effort from the processing unit than classical fuzzy PID.

In this paper, the proposed control scheme uses three LFPID controllers; each controller is responsible for maintaining a range of movement-controlled variables (i.e. roll, pitch, and yaw) at their respective user-desired values. The mathematical model of a quadrotor, used to design the proposed controller, is derived based on the Lagrange approach.

To perform the design of the controllers in a more realistic environment, the processor in the loop method (PIL) technique is used in this work. The PIL technique is used to test and validate the proposed control scheme. In the PIL set up, the controllers are implemented in the STM32F407 microcontroller; these latter will target the mathematical model of a quadrotor simulated in a separate MATLAB/Simulink environment. Data transfer between the hardware and software is via serial communication converter. This paper is organized as follows: The next section offers a brief presentation on the mathematic model of a quad-rotor and its topology. The third section describes the control strategy used. The fourth section presents the obtained simulation and validation results.

\section{RESEARCH METHOD}

In this section, the nonlinear dynamics model and the algoritme of control is presented.

\subsection{Quadrotor mathematical model}

Figure 1 presents the basic topology of the UAV (quadrotor). To move in the different axes of motion, the quadrotor uses four propellers attached to motors placed around the main body. The rotational speeds of the four rotors are used to control the pitch, roll, and yaw attitude of the vehicle. In this work, essentials of quadrotor model dynamic are discussed. Details on the behavior and general dynamics of four propellers unmanned aerial vehicle can be found in large numbers of papers [17]-[21].

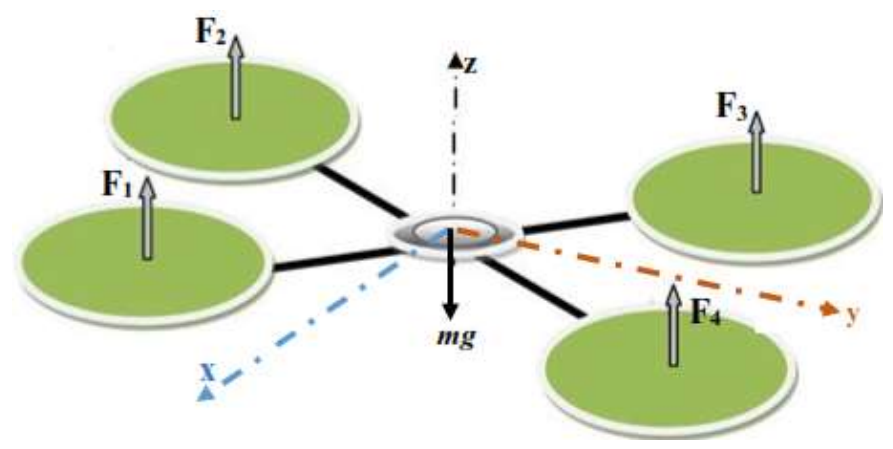

Figure 1. Quad-rotor axis system

Figure 2 shows the different axes of motion of a quadrotor. Quadrotor's mathematical model used, in this work, is based on the Lagrange approach and details explanation of its development is given in [22]-[26]. We consider an inertial frame and a body-fixed frame whose origin is in the center of mass of the quad-rotor as depicted in Figure 1. The different movement angles are defined as follows:

- Roll angle $\phi$ around vector $x$.

- Pitch angle $\theta$ around vector $y$.

- Yaw angle $\psi$ around vector $z$.

In the derivation of the mathematical model, the following assumptions are made: 1) the center of mass of the vehicle and the body-fixed frame origin coincide, 2) the structure is rigid and symmetrical, 3) the propellers are rigid in the plane and 4) Maneuvers are at small angles.

The dynamic of the quadrotor can be summarized in the following system of equations: 


$$
\left\{\begin{array}{c}
\ddot{\phi} I_{x}=J_{\text {rotor }} \dot{\theta}\left(\omega_{1}+\omega_{3}-\omega_{2}-\omega_{4}\right)+\left(I_{y}-I_{z}\right) \dot{\theta} \dot{\psi}+b l\left(\omega_{1}^{2}+\omega_{2}^{2}-\omega_{3}^{2}-\omega_{4}^{2}\right) \\
\ddot{\theta} I_{y}=J_{\text {rotor }} \dot{\phi}\left(-\omega_{1}-\omega_{3}+\omega_{2}+\omega_{4}\right)+\left(I_{z}-I_{x}\right) \dot{\phi} \dot{\psi}+b l\left(-\omega_{1}^{2}+\omega_{2}^{2}+\omega_{3}^{2}-\omega_{4}^{2}\right) \\
\ddot{\Psi} I_{z}=d\left(\omega_{1}^{2}+\omega_{3}^{2}-\omega_{2}^{2}-\omega_{4}^{2}\right)+\left(I_{x}-I_{y}\right) \dot{\theta} \dot{\phi}
\end{array}\right.
$$

where;

$b: \quad$ Thrust coefficient.

$d$ : Drag coefficient.

$J_{\text {rotor: }}$ Rotor Inertia.

$\omega_{i:} \quad$ Angular velocity of motor i.

$l: \quad$ Distance between the axis of rotation of the rotor and the center of the mass.
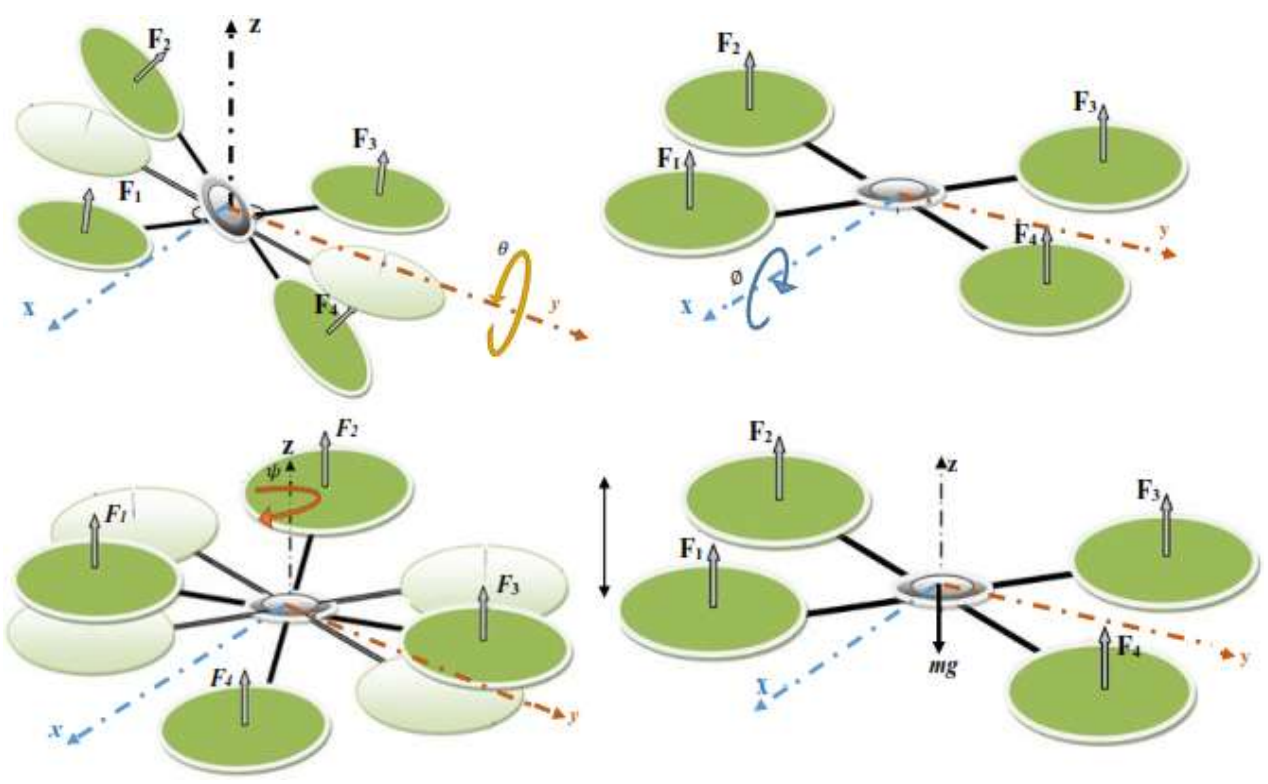

Figure 2. Flight mode of quad-rotor

\subsection{Proposed control scheme}

Figure 3 presents the proposed control scheme for the UAV. The robot is guided according to four references (desired values): the dynamics of vertical position reference $Z^{*}$, the roll reference $\phi^{*}$, the pitch $\theta^{*}$, and the yaw angle reference $\psi^{*}$. As shown in Figure 3, the feedback signals obtained from the system are compared to the yaw $\psi^{*}$, pitch $\theta^{*}$, and the roll $\phi^{*}$ setpoints. The resulting error signals are then fed to a lookup table based fuzzy logic PID controllers (LFPID). The simulated quadrotor dynamics block is built using the system of (1) and the motor-reducer actuators are simulated as first-order transfer functions.

Figure 4 shows the general topology of the LFPID. It has to inputs and one output. The inputs are respectively the error signal ' $E$ ' and the change of this error ' $C E$ '. We call 'error', the difference between reference and feedback signal. The output is a single signal which is the sum of the proportional integral and the derivative actions. As shown in Figure 4, the controller topology is almost identical to that of a fuzzy PID (FPID) control structure, but instead of using a conventional inference system, LFPID uses a pre-calculated logic stored in a 2D lookup table. The advantages of the LFPID algorithm are that it requires less computation as compared with FPID and achieves almost the same control performance as FPID.

To prove that FPID and LFPID have almost the same performance, both controllers were used to control the simulated mathematical model of the quadrotor, the results are presented in Figure 5. Figure 5 (left) shows time responses to the step-change in set point of Roll angle from $0^{\circ}$ to $8^{\circ}$ at $t=5 \mathrm{~s}$ with applying of wind perturbation of $0.05 \mathrm{~N} . \mathrm{m}$ at $t=15 \mathrm{~s}$. The two responses are, respectively, of the roll movement using the conventional FPID controller $\left(\phi_{F P I D}\right)$ and the roll movement using LFPID controller $\left(\phi_{L F P I D}\right)$. As shown, both controllers achieve almost the same performance. This is demonstrated by Figure 5 (right), which shows that the difference between the two output-time responses (i.e. $\phi_{F P I D}-\phi_{L F P I D}$ ) which is negligible. 


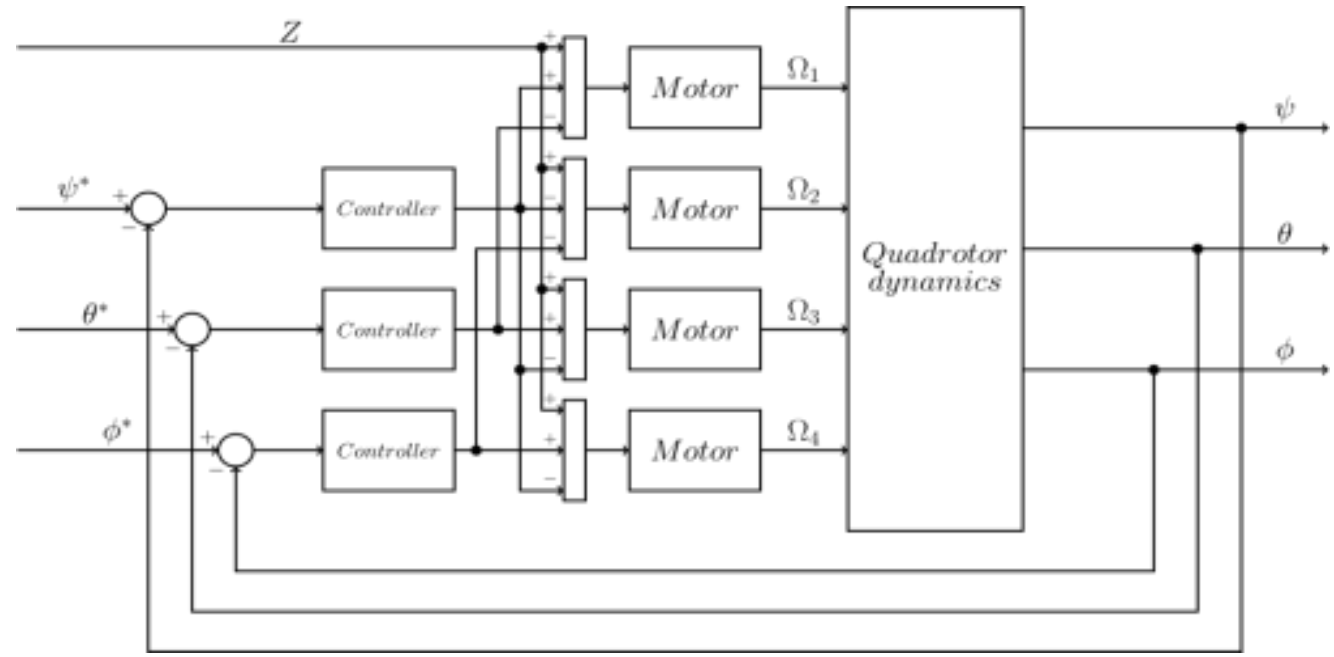

Figure 3. Block diagram of the proposed control scheme

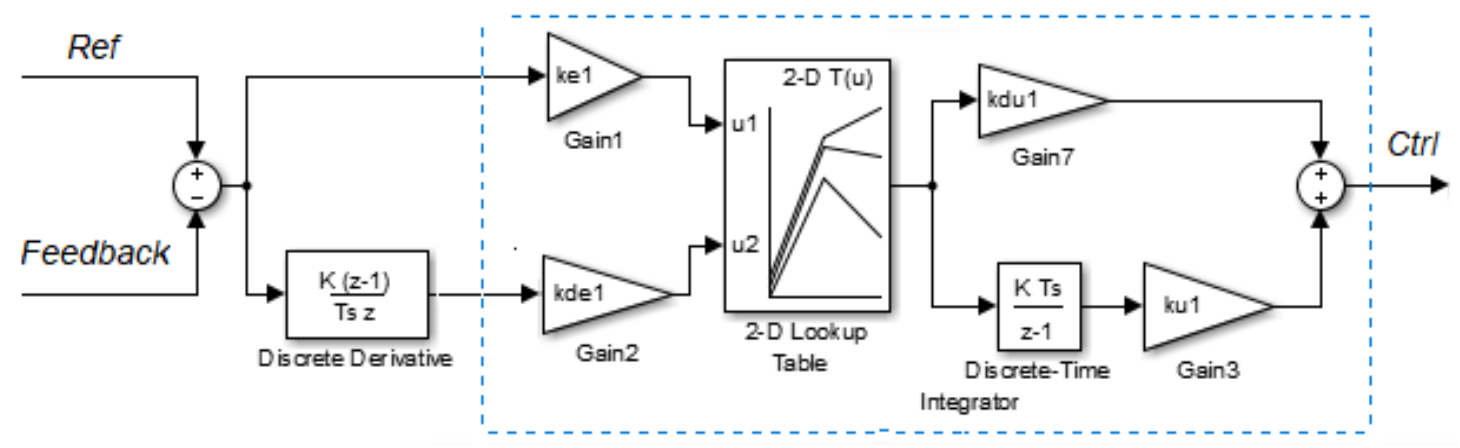

Figure 4. Block diagram of a 2D lookup Fuzzy PID controller
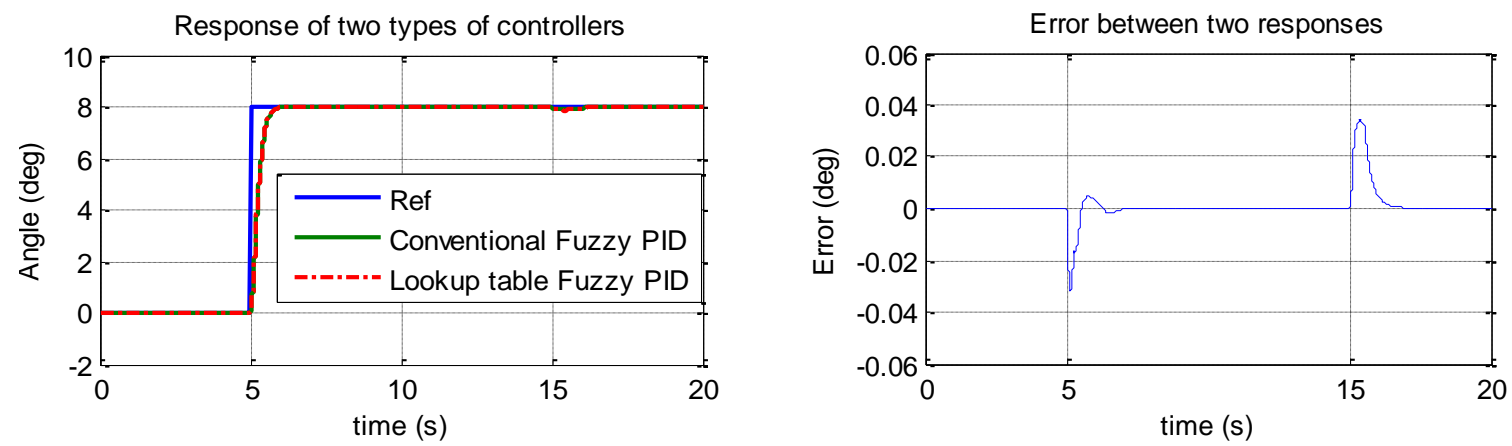

Figure 5. Difference between conventional and lookup table based fuzzy PID controller (roll movement)

The pre-calculate process or the construction of the lookup table is based on the behavior of the fuzzy inference system. This latter, in our work, having the error ' $E$ ' and the change of this error 'CE' as inputs and the control signal ' $U$ ' as output defined according to the table of rules shown in Table 1. Note that our choice fell on 5 membership function, for each variable, having the forms shown in Figure 6. 


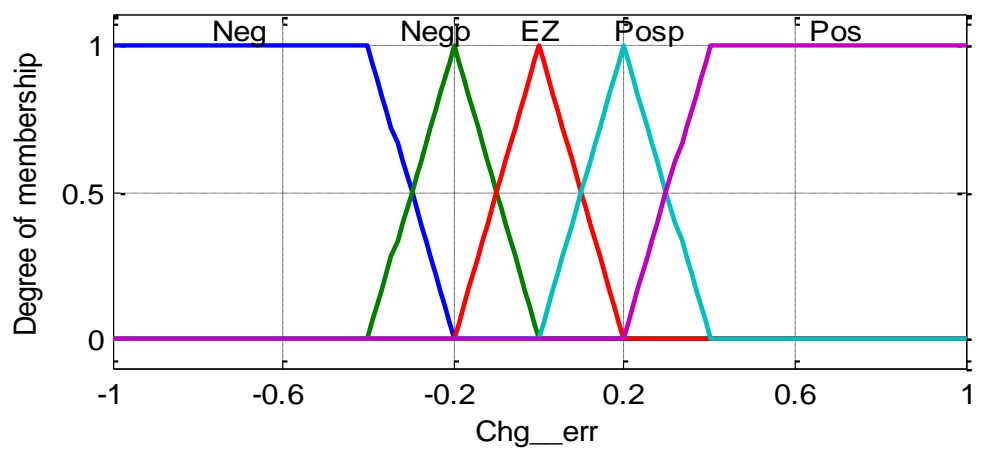

Figure 6. Membership functions

Table 1. Rules for fuzzy control

\begin{tabular}{lcccccc}
\hline & & \multicolumn{7}{c}{ Error ' $E$ ' } \\
& & Neg & Negp & EZ & Posp & Pos \\
\hline Change of Error CE & Neg & Neg & Neg & Neg & Nogp & EZ \\
& Negp & Neg & Neg & Nogp & EZ & posp \\
& EZ & Neg & Nogp & EZ & posp & Pos \\
& Posp & Negp & EZ & posp & Pos & Pos \\
& Pos & EZ & posp & Pos & Pos & Pos \\
\hline
\end{tabular}

\section{RESULTS AND DISCUSSION}

We present in this section, the simulation and the experimental results in order to valid the proposed control. To design and evaluate the performance of the proposed control scheme, simulation studies based on MATLAB/Simulink platform were first conducted using the model of quadrotor under different operating conditions including difficult situation with internal and external perturbations.

Then, the processor in the loop technique was used to test and validate the designed LFPID control scheme. This latter was implemented on an STM32F407 microcontroller that will target the simulated mathematical model of the quadrotor as a MATLAB/Simulink program within a computer. The data transfer between computer and microprocessor is managed by a serial converter. Figure 7 shows the experimental set up of the processor in the loop system.

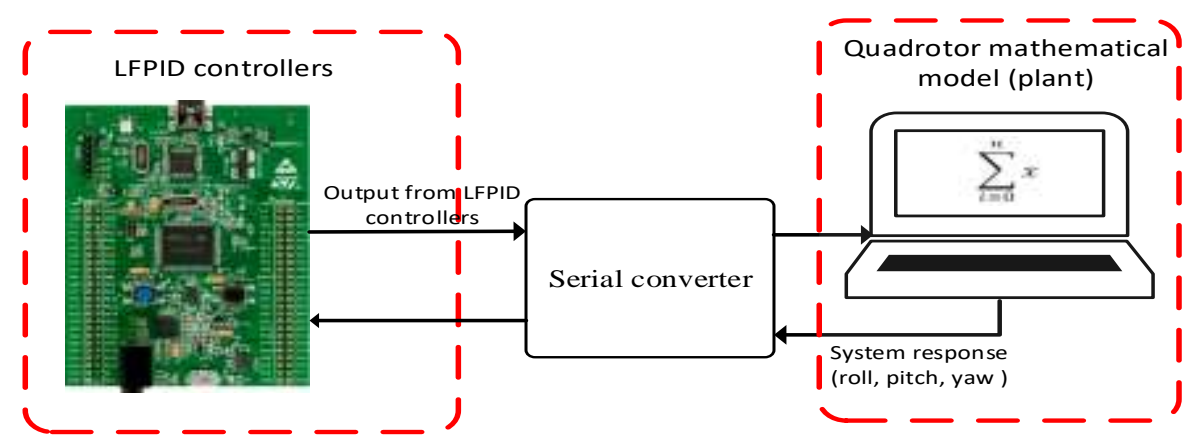

Figure 7. Block diagram of PIL

Figures 8-10 show simulation and PIL results for reference changes for the three control algorithms PID, FPID, and LFPID. In this study, a PID controller is used to our system to compare its performance with the proposed LFPID controller. In Figure 8, we show the behavior of the three correctors (PID, FPID and LFPID) with respect to changes in the set points of the three angles (roll, pitch and yaw). It is clear that we have formed a complex template where the angle changes are not made separately, reflecting realistic maneuvers.

This Figure 8 shows that the performance of the proposed approach is significantly higher than that of the conventional PID corrector; Total absence of overshoots for all situations and a very fast response time (equivalent to $0.9 \mathrm{~s}$ ) compared to that of the PID which is about $7.3 \mathrm{~s}$ 
Keeping the same template, the rest of the Figures 9 and 10, detailing the different angles, are obtained when placing the drone in unfavorable conditions:

- Application of external moments such as a gust of wind blowing at different times:

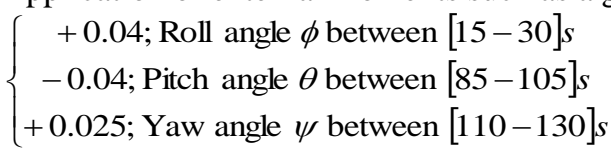

- Applying an internal perturbation by increasing all the inertia (Ix, Iy and Iz) by $100 \%$ in initial conditions

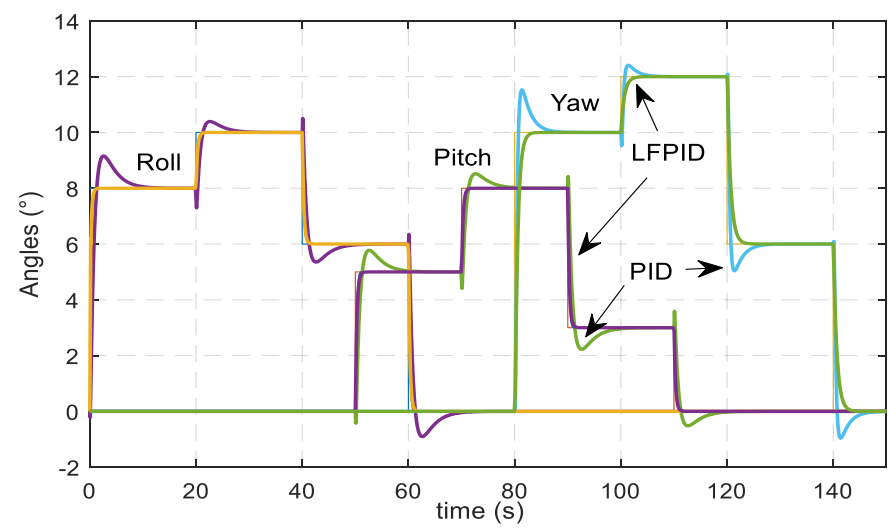

Figure 8. Simulation with PIL results of quadrotor attitude control
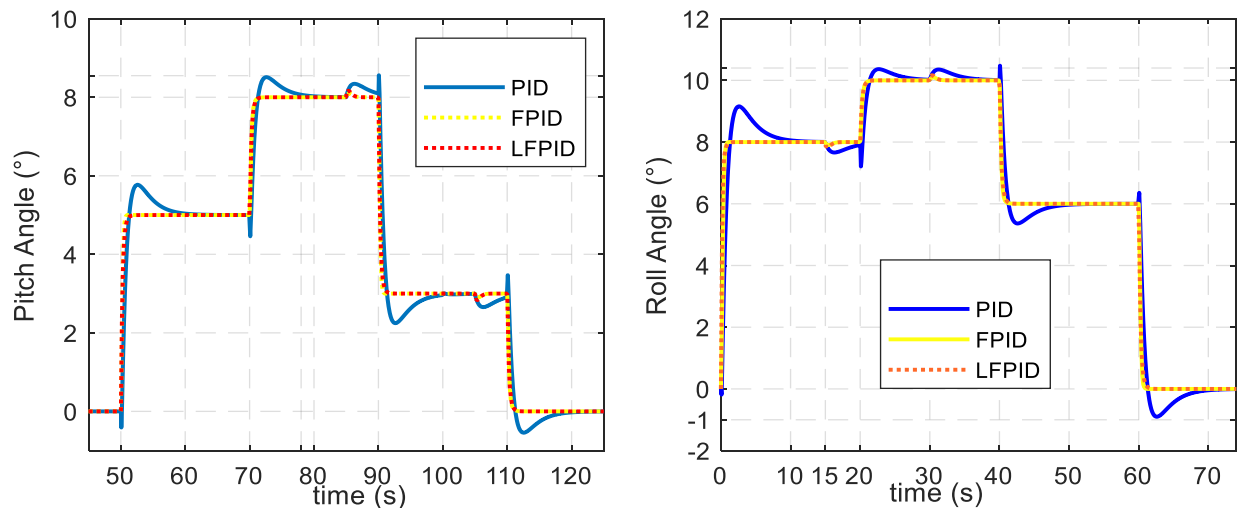

Figure 9. Quadrotor behavior with internal \& external perturbation (Roll \& Pitch angle)

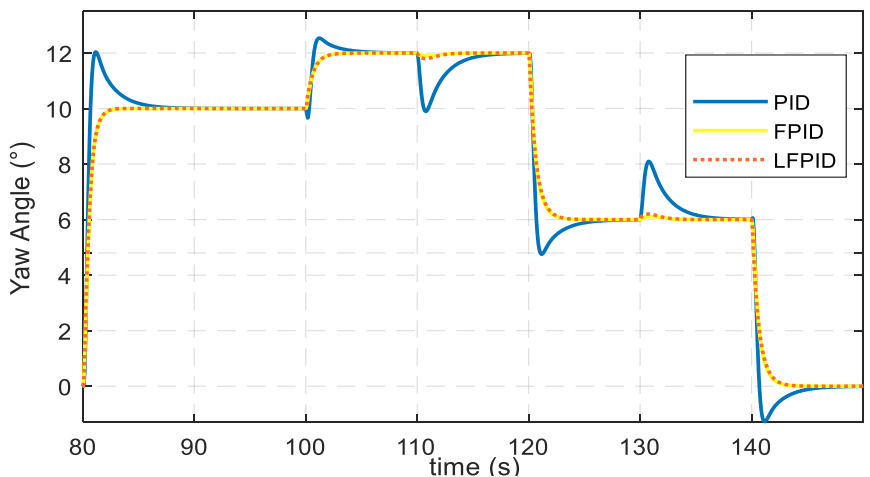

Figure 10. Quadrotor behavior with internal \& external perturbation (Yaw angle) 
The results reveal a robust behavior of the lookup table based on fuzzy PID control for both internal and external disturbances. Table 2 summarizes the quantitative analysis of behavior using the two approaches (We note that the manevers are at small angles). The designed LFPID is tested and validated using the PIL approach. This is confirmed by the perfect matching of time responses of movement variables, shown in all figures, of quadrotor closed-loop control using FPID simulated in MATLAB/Simulink and LFPID implemented in STM32F407 microcontroller.

Table 2. Quantitative analysis of the results

\begin{tabular}{lcccc}
\hline & Roll \& Pitch & \multicolumn{2}{c}{ Yaw } \\
& LFPID & PID & LFPID & PID \\
\hline Start-up overrun & 0 & $1^{\circ}$ & 0 & $2^{\circ}$ \\
Overshoot when changing reference & 0 & $0.5^{\circ}$ & 0 & $1.2^{\circ}$ \\
Angle due to the application of the wind & $0.1^{\circ}$ & $0.35^{\circ}$ & $0.15^{\circ}$ & $2^{\circ}$ \\
Disturbance rejection time & $2 \mathrm{~s}$ & $8 \mathrm{~s}$ & $3 \mathrm{~s}$ & $8 \mathrm{~s}$ \\
\hline
\end{tabular}

\section{CONCLUSIONS}

In this paper, a lookup table based fuzzy PID (LFPID) controller was proposed to control a UAV system. The elements of the lookup table are calculated from a classical fuzzy inference system. The purpose of using an LFPID is to reduce the computational burden on the processing unit (MCU) while maintaining the same performance that a conventional fuzzy controller provides. Simulation results were validated by using a processor in the loop technique. Both results (simulation/PIL) proved the validity of the proposed controller.

\section{REFERENCES}

[1] A. Noordin, M. A. M. Basri, and Z. Mohamed, "Simulation and experimental study on PID control of a quadrotor MAV with perturbation," Bulletin of Electrical Engineering and Informatics, vol. 9, no.5, pp.1811-1818, 2020, doi: 10.11591/eei.v9i5.2158.

[2] N. A. S. Putro, A. Dharmawan, and T. K. Priyambodo, "Quadrotor Control System with Hand Movement Sign as an Alternative Remote Control, "International Journal of Robotics and Automation (IJRA), vol. 6, no. 2, pp. 131-140, 2017, doi: 10.11591/ijra.v6i2.pp131-140.

[3] V. P. Kharchenko, N. S. Kuzmenko, and I. V. Ostroumov, "Identification of unmanned aerial vehicle flight situation," 2017 IEEE 4th International Conference Actual Problems of Unmanned Aerial Vehicles Developments (APUAVD), Kiev, Ukraine, 2017, pp. 116-120, doi: 10.1109/APUAVD.2017.8308789.

[4] V. Burnashev and A. Zbrutsky, "Control Loops Synthesis of a Supersonic Unmanned Aerial Vehicle," 2019 IEEE 5th International Conference Actual Problems of Unmanned Aerial Vehicles Developments (APUAVD), Kiev, Ukraine, 2019, pp. 102-105, doi: 10.1109/APUAVD47061.2019.8943872.

[5] V. Tyurin, O. Martyniuk, V. Mirnenko, P. Open'ko, and I. Korenivska, "General Approach to Counter Unmanned Aerial Vehicles," 2019 IEEE 5th International Conference Actual Problems of Unmanned Aerial Vehicles Developments (APUAVD), Kiev, Ukraine, 2019, pp. 75-78, doi: 10.37105/sd.36.

[6] Y. Cheng, S. Xu, Z. Huang, B. Xiong, and C. Wang, "Stability control of small fixed-wing UAV," Proceeding of the 11th World Congress on Intelligent Control and Automation, Shenyang, China, 2014, pp. 1056-1060, doi: 10.1109/WCICA.2014.7052863.

[7] O. P. Krivonosenko and O. V. Savchenko, "Synthesis of optimal structure of UAV stabilization system by full-scale experiment data," 2015 IEEE International Conference Actual Problems of Unmanned Aerial Vehicles Developments (APUAVD), Kyiv, UKraine, 2015, pp. 112-115, doi: 10.1109/APUAVD.2015.7346575.

[8] A. H. Ginting, O. Wahyunggoro, and A. I. Cahyadi, "Attitude Control of Quadrotor Using PD Plus Feedforward controller on SO(3)," International Journal of Electrical and Computer Engineering (IJECE), vol. 8, no. 1, pp. 566-577, 2018, doi: 10.11591/ijece.v8i1.pp566-575.

[9] J. M. Valenzuela, R. P. Alcocer, M. G. Medina, and A. Dzul, "Nonlinear PID-Type Controller for Quadrotor Trajectory Tracking," in IEEE/ASME Transactions on Mechatronics, vol. 23, no. 5, pp. 2436-2447, 2018, doi: 10.1109/TMECH.2018.2855161.

[10] D. Kucherov, A. Kozub, and A. Rasstrygin, "Setting the PID Controller for Controlling Quadrotor Flight: a Gradient Approach," 2018 IEEE 5th International Conference on Methods and Systems of Navigation and Motion Control (MSNMC), Kiev, Ukraine, pp. 90-93, 2018, doi: 10.1109/MSNMC.2018.8576294.

[11] I. G. N. A. Indra Mandala, Franky, and Y. Y. Nazaruddin, "Optimization of Two Degree of Freedom PID Controller for Quadrotor with Stochastic Fractal Search Algorithm," 2019 IEEE Conference on Control Technology and Applications (CCTA), Hong Kong, China, 2019, pp. 1062-1067, doi: 10.1109/CCTA.2019.8920540.

[12] H. Glida, L. Abdou, and A. Chelihi, "Optimal Fuzzy Adaptive Backstepping Controller for Attitude Control of a Quadrotor Helicopter," 2019 International Conference on Control, Automation and Diagnosis (ICCAD), Grenoble, France, 2019, pp. 1-6. 
[13] V. P. Tran, F. Santoso, M. A. Garratt, and I. R. Petersen, "Adaptive Second-Order Strictly Negative Imaginary Controllers Based on the Interval Type-2 Fuzzy Self-Tuning Systems for a Hovering Quadrotor with Uncertainties," in IEEE/ASME Transactions on Mechatronics, vol. 25, no. 1, pp. 11-20, Feb. 2020, doi: 10.1109/TMECH.2019.2941525.

[14] T. Huang, D. Huang, and D. Luo, "Attitude Tracking for a Quadrotor UAV Based on Fuzzy PID Controller," 2018 5th International Conference on Information, Cybernetics, and Computational Social Systems (ICCSS), Hangzhou, China, 2018, pp. 1-6, doi: 10.1109/ICCSS.2018.8572353.

[15] Q. Jing, Z. Chang, H. Chu, Y. Shao, and X. Zhang, "Quadrotor Attitude Control Based on Fuzzy Sliding Mode Control Theory," 2019 Chinese Control Conference (CCC), Guangzhou, China, 2019, pp. 8360-8364.

[16] H. E. Glida, L. Abdou, A. Chelihi, and C. Sentouh, "Optimal Direct Adaptive Fuzzy Controller based on Bat Algorithm for UAV Quadrotor," 2019 8th International Conference on Systems and Control (ICSC), Marrakesh, Morocco, 2019, pp. 52-57, doi: 10.1177/09544100211025379.

[17] J. de Jesus Rubio, J. Humberto Perez Cruz, Z. Zamudio, and A. J. Salinas, "Comparison of two quadrotor dynamic models," in IEEE Latin America Transactions, vol. 12, no. 4, pp. 531-537, June 2014, doi: 10.1109/TLA.2014.6868851.

[18] P. Patel and J. Dave, "Design and Dynamic Modelling of Quadrotor VTOL aircraft," 2020 4th International Conference on Electronics, Communication and Aerospace Technology (ICECA), Coimbatore, India, 2020, pp. 105-112.

[19] M. Walid, N. Slaheddine, A. Mohamed, and B. Lamjed, "Robust internal model controller for quadrotor UAV," 2018 International Conference on Advanced Systems and Electric Technologies (IC_ASET), Hammamet, Tunisia, 2018, pp. 138-143.

[20] P. Lyu, J. Lai, J. Liu, L. Zhang, and S. Liu, "A novel integrated navigation system based on the quadrotor dynamic model," 2018 IEEE/ION Position, Location and Navigation Symposium (PLANS), Monterey, CA, USA, 2018, pp. 688-695.

[21] A. M. A. M. Basri and A. Noordin, "Optimal backstepping control of quadrotor UAV using gravitational search optimization algorithm, " Bulletin of Electrical Engineering and Informatics, vol. 9, no. 5, pp.1819-1826, 2020, doi: 10.11591/eei.v9i5.2159.

[22] A. Benbouali, R. Taleb, F. Chabni, and T. Bessaad, "PI control of quad-rotor unnamed vehicle based on lagrange approach modelling," 2016 8th International Conference on Modelling, Identification and Control (ICMIC), Algiers, Algeria, 2016, pp. 1-4.

[23] B. Halc1, V. Gazi, and O. Cihan, "Modelling and Coordination of a Swarm of Quadrotors Using Lagrange Dynamics and Potential Functions," 2019 24th IEEE International Conference on Emerging Technologies and Factory Automation (ETFA), Zaragoza, Spain, 2019, pp. 963-970, doi: 10.1109/ETFA.2019.8869248.

[24] A. Aksjonov, V. Vodovozov, and E. Petlenkov, "Sensorless control of the three-dimensional crane using the Euler-Lagrange approach with a built-in state-spaee model," 2015 56th International Scientific Conference on Power and Electrical Engineering of Riga Technical University (RTUCON), Riga, Latvia, 2015, pp. 1-4.

[25] Oyetola O. K. and Olaluwoye O. O., "Simulink and Simelectronics based Position Control of a Coupled Mass-Spring Damper Mechanical System," International Journal of Electrical and Computer Engineering (IJECE), vol. 8, no. 5, pp. 3636-3646, 2018.

[26] A. J. Moshayedi, M. Gheibollahi, and L. Liao, "The quadrotor dynamic modeling and study of metaheuristic algorithms performance on optimization of PID controller index to control angles and tracking the route," International Journal of Robotics and Automation (IJRA), vol. 9, no. 4, pp. 256-270, 2020. 